\title{
GRÜNDE FÜR DAS SCHEITERN DER AFRIKANISCHEN OPPOSITION IN SÜDAFRIKA
}

\author{
Von Denis Nkhwazi
}

Über das politische System Südafrikas ist sehr viel geschrieben worden - sowohl akademische als auch journalistische Arbeiten. Die meisten dieser Arbeiten beschäftigen sich mit der Philosophie und der Politik der Apartheid, der Art des südafrikanischen Rassismus. In der Hauptsache beschreiben diese Studien die politische Ideologie der Buren, die Geschichte ihres Aufstieges zur Macht, die inneren Auseinandersetzungen der Burengemeinschaft, die Beziehungen zwischen den Buren und der englischen Volksgruppe und die Methoden, mit denen die südafrikanischen Regierungen es verstanden haben, die schwarze Mehrheit von jeglicher politischen Partizipation auszuschließen. Außerdem haben einige Autoren die Lage der Afrikaner untersucht, die meisten von ihnen von einem symphatisierenden Standpunkt aus, so zum Beispiel Colin Legum, Anthony Sampson, Mary Benson, Brian Bunting, Freimut Duve und viele andere ${ }^{1}$. In allen diesen Arbeiten dominiert die Unterstützung für die afrikanische Sache. Die südafrikanische Regierung und das weiße Establishment haben alle Informations- und Propagandamedien zu ihrer Verfügung, um ihren Standpunkt bei den Regierungen der westlichen Welt durchzusetzen. Die Afrikaner haben nicht diese Möglichkeit. Außerdem reicht das Wirtschaftsund Industriepotential Südafrikas dazu aus, die Freundschaft Westeuropas und Nordamerikas zu gewinnen. Aus diesen Gründen befinden sich die Afrikaner, was die Information angeht, in einer sehr ungünstigen Lage. Auch die zahlreichen, völlig unkritischen afrophilen Arbeiten können diese Informationslücke nicht ausfüllen. Sämtliche Veröffentlichungen dieser Autoren wollen internationale Sympathie und Anerkennung für den afrikanischen Freiheits- und Unabhängigkeitskampf erheischen. Das Bemühen, internationale Unterstützung zu erlangen, hat jedoch dazu geführt, daß viele fundamentale Fragen ausgeklammert wurden.

Wie ist es zum Beispiel zu erklären, daß sich in den Nachbarstaaten Südafrikas wie Sambia, Malawi und Tanzania gut organisierte politische Parteien bildeten, während die schwarzen Südafrikaner es nicht rerstanden, sich in dieser Weise zu organisieren? Ist diese Unfähigkeit wirklich auf die überwältigende Macht der Regierung und der Polizei zurückzuführen? Wenn es den Vietnamesen gelingt, die Amerikaner jahrelang in Schach zu halten, warum vermögen dann die 15 Millionen Schwarzen in Südafrika nicht, auf die nur 3 Millionen Weiße in ähnlicher Weise einzuwirken? Sind sie mit den Lebensbedingungen dort zufrieden? Haben die Schwarzen die besonderen sozialen Verhältnisse in Südafrika anerkannt? Wenn das der Fall ist, haben sie die notwendigen Konsequenzen daraus gezogen? Edward Feit hat in seinem Buch "African Oppositions in South Africa"2, den mutigen Versuch unternommen, auf diese Fragen eine Antwort zu finden. In seiner Arbeit „Südafrika, Soziologie einer Rassengesellschaft", hat Heribert Adam die Argumentation von Feit noch ergänzt ${ }^{3}$. Die vorliegende Arbeit soll einen Beitrag zur kritischen Auseinandersetzung mit der Widerstandsbewegung in Südafrika leisten.

1 Siehe: Legum, Colin \& Margaret, The South African Crisis for the West, London 1964; Sampson, A., The Treason Cage, London, 1958; Benson, M., The African Patriots, London, 1963; Bunting, B. The Rise of the South African Reich London, 1964; Duve, F., (ed.), Kap ohne Hoffnung oder die Politik der Apartheid, Reinbek bei Hamburg, 1965.

2 Siehe: Feit, Edward, African Oppositions in South Africa, Stanford, 1967.

3 Siehe: Adam, H., Südafrika, Soziologie einer Rassengesellschaft, Frankfurt, 1969. 


\section{Die Opposition der nicht repräsentierten Mehrheit}

Das politische, wirtschaftliche und soziale System Südafrikas ist ein Kastensystem, das sich aus drei Hauptgruppen, nämlich den Buren, Engländern und Afrikanern zusammensetzt. Die Inder und die Farbigen (Mischlinge) bilden eigene Gruppen. Es ist gleichzeitig ein rassisches System, weil die ganze politische Macht von einer bestimmten rassischen Gruppe kontrolliert wird. Das System, dessen Erhaltung der rassischen Schichtung ein systematisch unausweichliches primäres Objekt der Politik bildet, ist einzigartig. Wie in keinem anderen politischen System der Welt ist das politische und wirtschaftliche System daraufhin organisiert, daß jegliche Integration $\mathrm{zwischen}$ den verschiedenen Gruppen unmöglich gemacht wird ${ }^{4}$.

Obgleich in allen Bereichen der wirtschaftlichen Betätigung die Nichtweißen mitwirken, ist die politische und wirtschaftliche Kontrolle des Landes ausschließlich in den Händen der Europäer. Mit anderen Worten partizipieren alle Angehörigen der Bevölkerungsgruppen an dem Wirtschaftsprozeß und sind von dessen Ergebnis abhängig, während ihr Schicksal und ihre Beteiligung an der Wohlfahrt des Staates von ihrer Rasse bestimmt wird. Südafrika ist ein Staat, in dem Mitglieder der verschiedenen Rassen selten zusammentreffen, außer in dem Abhängigkeitsverhältnis zwischen einem weißen Arbeitgeber und einem schwarzen Arbeitnehmer. Angehörige der jeweiligen Rassengruppen wohnen in verschiedenen Gebieten, gehen zu verschiedenen Kirchen und besuchen verschiedene Schulen, benutzen verschiedene Verkehrsmittel (auch verschiedene Flugzeuge), werden aus verschiedenen Postschaltern bedient, sitzen auf verschiedenen Parkbänken, essen in verschiedenen Restaurants, liegen in verschiedenen Krankenhäusern (außer im Falle einer Herzverpflanzung, wenn ein Schwarzer als Stifter in aller Eile ins weiße Krankenhaus gebracht wird), und wenn sie sterben, werden ihre Leichen in verschiedenen Wagen und nach verschiedenen Friedhöfen transportiert ${ }^{5}$.

In einem Zeitalter der Geschichte, in dem rassische Unterdrückung allgemein als moralisch unakzeptabel angesehen wird, gilt die südafrikanische Situation als einzigartig. Demzufolge ist Südafrika heute ein isoliertes und bedrohtes Land. Diese Bedrohung oder diese Opposition ist sowohl innerhalb als auch außerhalb zu spüren, denn solange das System der Apartheid existiert, werden die neuen afrikanischen Staaten, die Unabhängigkeitsbewegung als nicht vollendet betrachten ${ }^{6}$.

\section{Die politischen Zielsetzungen der internen Massenopposition}

In einem Kastensystem mögen politische Oppositionen verschiedene Formen annehmen und verschiedene Ziele und Methoden zugrunde legen. Bei der Analyse des afrikanischen Nationalismus in Südafrika sollte im Auge behalten werden, daß man es hier mit der Opposition einer Gemeinschaft zu tun hat, die über keine politische Repräsentation im Parlament verfügt. Die „site of action“ dieser Opposition muß also notwendigerweise irgendwo anders liegen als in den staatlichen Institutionen. Ihre Strategie, Organisation und Wirkungsmöglichkeiten sind durch den gegebenen politischen Kontext bedingt. Die unmittelbare Frage bei einer Untersuchung der Opposition in einem Kastensystem (besonders wenn die oppo-

4 Vgl. Thompson, L. M., The Republic of South Africa, Boston, 1966, S. 2.

5 Vgl. Ibid, S. 2.

6 Vgl. Ibid, S. 2 ff. 
nierende Gruppe die Mehrheit bildet), ist die Frage der Zielsetzung dieser Opposition. Leonard Thompson hat den afrikanischen Freiheitskampf in drei wesentlichen Entwicklungsphasen klassifiziert. Mit Hilfe dieser Phasenperiodisierung sollen die Absichten der afrikanischen Opposition erklärt werden? ${ }^{7}$ Die Periode von der Gründung des „African National Congress“ (ANC) im Jahre 1912 bis 1948 wird als Reform-Phase bezeichnet. In dieser Zeitspanne beschäftigte sich der ANC hauptsächlich mit Bestrebungen für Reformen bestimmter Teile der Regierungspolitik. Dabei wurde die politische Struktur Südafrikas nicht angegriffen. Die Zeit von 1948-1960 und von 1960 bis heute bildet eine weitere Phase, die als "revolutionary in objective“ bezeichnet wird8. Diese Klassifikation wäre in Ubereinstimmung mit politischen Oppositionen gewesen, die in dem Dahlschen Modell "revolutionary movements“ genannt werden. Sie haben das Ziel, das Regierungspersonal, die allgemeine spezifische Regierungspolitik und die politische und ökonomische Struktur des Landes zu ändern, anders gesagt: das ganze politische System umzuwandeln ${ }^{9}$. Dahls Modell (das man auf westliche Demokratien beschränken sollte) und das für die Erklärung der südafrikanischen schwarzen Opposition hier von Thompson anscheinend übernommen wurde, ist nur mit Einschränkungen verwendbar. Die oben erwähnte völlige Umgestaltung der politischen Struktur würde im Falle Westeuropas zweifellos eine Revolution des politischen Systems bedeuten mit Sozialismus oder Kommunismus als Endergebnis. Wenn er von einer Umgestaltung des politischen Systems spricht, denkt Dahl sehr wahrscheinlich an die Liquidierung der parlamentarischen Institutionen und des Marktpreissystems. Versucht man diese Gedanken auf die afrikanische Opposition in Südafrika anzuwenden, so erweist es sich als notwendig, einige Modifikationen vorzunehmen. In erster Linie scheint diese Konfusion in der Klassifikation auf einem Mißverständnis der Zielsetzungen afrikanischer Unabhängigkeitsbewegungen zu beruhen. Sicher würde die Erlangung politischer Rechte der Afrikaner in Südafrika zu einer total neuen Besetzung der Regierung und damit zu neuen Richtlinien der Politik führen. Aber selbst diese Änderung brauchte keine unausweichliche Zerstörung der vorhandenen politischen und sozio-ökonomischen Struktur des Landes herbeizuführen. Die Erfahrungen mit den meisten selbständigen afrikanischen Staaten haben gerade gezeigt, daß die einzige bezeichnende Änderung vom kolonialen System nur das Mehrheitsprinzip gewesen ist. Die politischen und wirtschaftlichen Institutionen, wie z. B. das Parlament, die Justiz, das freie Marktsystem und von noch wichtigerer Bedeutung, die allgemeine intellektuelle Orientierung, bleiben ungestört wie zuvor. Die Machtübernahme durch die Mehrheit kann nicht selbst als „revolutionary“ bezeichnet werden. Es wird sich vielmehr nur um eine Übertragung der politischen Macht von einer rassischen Minderheit zu einer Mehrheit handeln - eine Mehrheit, die dieselben politischen Institutionen besetzt und dieselbe wirtschaftliche Praxis fortsetzen wird. Der einzige Unterschied wird der, daß statt der Weißen die Schwarzen zum Herrscher werden. Das zweite Problem scheint in der Verwechslung von Zielsetzung und Methoden zu liegen. Die afrikanische Freiheitsbewegung ist sicher in den letzten Jahren dazu gezwungen worden, revolutionäre Methoden zu gebrauchen, beispielsweise die Anwendung von Gewalt. Aber selbst der Sturz einer Regierung durch Gewalt (wenn durch die Regierungs-

\footnotetext{
7 Vgl. Ibid, S. 165.

9 Vgl. Dahl, R. A. (ed.) Patterns of Oppositions, in: Political Oppositions in Western Democracies, New Haven-London, 1966, S. 332 ff.
} 
maßnahmen alle übrigen friedlichen Wege aussichtslos gemacht werden) bedeutet nicht, daß radikale politische und wirtschaftliche Änderungen herbeigeführt werden. Während des Rivonia-Prozesses am 20.April 1964 hat Nelson Mandella das kommunistische System mit klaren Worten abgelehnt. Gleichzeitig bekräftigte er seine Bewunderung und Hochachtung für das parlamentarische System und für das Rechtswesen nach dem Bilde Englands. „Under socialism the workers hold state power. They and the peasantry own the means of production, the land ... All production is for use and not for profit. The Charter (der ANC) does not contemplate such profound economic and political changes ${ }^{10}$ " Außerdem entspricht die Bezeichnung der Klassifikation einer Partei als revolutionär, die vom Nobelpreisträger Chief Luthuli geleitet wurde, und zu deren Führung einige der konservativsten, religiösen, bürgerlichen und traditionellen Elemente angehören, keiner Realität. Die südafrikanische Mehrheitsopposition ist nichts anderes als eine normale populäre Unabhängigkeitsmassenbewegung, die das maßvolle Ziel verfolgt, das Selbstbestimmungsrecht $\mathrm{zu}$ erlangen und die durch die bestehenden politischen Verhältnisse dazu gezwungen ist, revolutionäre Mittel zu gebrauchen ${ }^{11}$.

\section{Der „African National Congress“ als Opposition}

Seit 1912 bis zu seinem Verbot im Jahre 1960 war der ANC die bedeutendste politische Partei der Afrikaner Südafrikas. Ähnlich wie für die anderen NichtweiPen, ist es für die Schwarzen ungeheuer schwierig, sich politisch zu organisieren. Eins der großen Handicaps ist der enorme Unterschied zwischen der kleinen ausgebildeten Minorität und der Mehrheit der Afrikaner ohne Schulausbildung. Durch die immer größer werdende Zahl der in Industrie und Handel geschulten Berufstätigen wird diese Schwäche langsam beseitigt. Diese Tatsache dürfte als der bedeutendste Faktor in der Entwicklung der afrikanischen nationalistischen Bewegung gelten. Ein weiteres Problem stellen die zahlreichen Barrieren gegen die Abhaltung von politischen Konferenzen dar. Selbst um in den Reservaten eine politische Versammlung abzuhalten, benötigt man die Erlaubnis der weißen Administration. "The Suppression of Communism Act" ist ein weiteres effektives Mittel, um politische Führer in einsame, weitentfernte Gebiete zu sperren wie z. B. im Falle Chief Luthulis. Für eine außerparlamentarische Opposition können solche Maßnahmen der Regierung die Effektivität der Opposition erheblich herabsetzen. Beispielsweise wurde im November 1962 der Generalsekretär des ANC zusammen mit vielen anderen wenige Minuten vor Anfang der Jahresversammlung verbannt. Ferner soll die Position der südafrikanischen Polizei genannt werden. Der Geheimdienst wurde schon im Anfang der fünfziger Jahre ausgebaut. Es ist der Polizei sogar gelungen, ins Organisationsnetz des ANC einzudringen. Dadurch konnte die Regierung die Intentionen des ANC vorher kennen. Polizeirazzien in den Häusern der ANC-Funktionäre kamen häufig vor. Der ANC mußte praktisch unter kontinuierlicher Úberwachung des Sicherheitsdienstes arbeiten. Die Angst vor der Geheimpolizei entmutigte angehende Aktivisten des ANC. Als der ANC nach dem Verbot die Untergrundbewegung „Umkonto we Sizwe“ (Speer der Nation) gründete, scheiterten diese Bemühungen an der außerordentlich rücksichtslosen Effizienz

\footnotetext{
10 Mandela, N., Freedom in Our Life Time, in: First, R. (ed.), No Easy Walk to Freedom, London, 1965,

11 Vgl. Carter, G.M., The Politics of Inequality, London, 1962, S. $358 \mathrm{ff.}$
} 
des südafrikanischen Polizeiapparates. Selbst Luthuli wurde einmal der Ausweis auf dem Flugplatz abgenommen, um dadurch seine Teilnahme auf der „Western Areas Campaign" $\mathrm{zu}$ verhindern ${ }^{12}$.

Trotz der Tatsache, daß die nichteuropäischen Organisationen fast am Rande der politischen Illegalität operieren müssen, ist es den Afrikanern, den Asiaten und den Farbigen gelungen, politische und quasi politische Bewegungen zu gründen, z. B. Clement Kadalie's spektakuläre und dynamische Industrial and Commercial Workers' Union aus den zwanziger Jahren mit einer Rekordmitgliedschaft von 100 000. Erst während der Kampangne von 1952 konnte der ANC zu dieser Mitgliedsgröße anwachsen. Aber der heutige fast totale Niedergang der Industrial and Commercial Workers' Union zeigt die weiteren Schwierigkeiten der afrikanischen Organisationen: a) ihre Abhängigkeit von einer oder wenigen mächtigen Persönlichkeiten; b) die Tendenz führender Persönlichkeiten, größeren Wert auf persönliches und weniger auf Organisationsinteresse zu legen; c) die Schwierigkeit, ein systematisches Programm unter solchen einschränkenden Verhältnissen zu entwickeln; d) und die immer wachsenden Strafbedrohungen der Regierung.

\section{Die Frage der Übereinstimmung von Organisation und Strategie}

Nach der Verfassung des ANC von 1943 wurden die Zweigorganisationen als Grundlage der Parteiorganisation festgelegt, während die Aufsichtsfunktion den vier Provinzialkonferenzen und der Nationalkonferenz zugeschrieben wurde. Zur Exekutive der Partei wurde das „National Executive Committee“ ernannt. Die eigentliche administrative Funktion aber wurde von dem Working Committee durchgeführt. Obwohl nach der Verfassung die Funktion des zentralen "decision making" dem National Executive Committee zugedacht war, hat zweifellos das Working Committee diese Rolle übernommen. Aus finanziellen Gründen ist man zu dem Entschluß gekommen, daß Mitglieder des Working Committees aus den Persönlichkeiten rekrutiert wurden, die innerhalb $80 \mathrm{~km}$ des nationalen Hauptquartiers in Johannesburg ihren Wohnsitz hatten. Diese Regelung hat zur Folge, daß es unmöglich war, das Working Committee aus Mitgliedern des Vorstandes zu konstituieren. Die Organisation sollte von einem jährlichen Beitrag von 1,25 DM pro Mitglied finanziert werden. Die angegliederten Schwesternverbände sollten zwei Drittel ihrer Einkünfte der zentralen Parteiorganisation zufließen lassen. Die Kontrolle der Finanzen und die Formulierung administrativer Regelungen wurden dem Working Committee zugelegt. Ferner übte dieses Gremium auch quasi juristische Funktionen aus. Durch dieses Arrangement ist das Working Committee zum mächtigsten Organ der Partei geworden, und zwar auf Kosten der Verantwortung des Vorstandes. Aber es gibt noch andere Schwächen in der Verfassung. Die Verhältnisse zwischen den Jugend- und Frauenorganisationen und der Hauptbewegung ist nirgendwo definiert worden. Gerade für eine außerparlamentarische Opposition sind die Jugend- und Frauenorganisationen von enormer strategischer Bedeutung. Die von dieser Auslassung resultierende Konfusion verrät erstaunliche organisatorische Naivitä $\mathrm{t}^{13}$. Dieses Versäumnis, die Beziehungen zwischen der Jugendbewegung und der Mutterorganisation klar festzulegen, hat dazu geführt, daß die

$12 \mathrm{Vgl}$. Carter, G. M. a. a. O., S. $358 \mathrm{ff}$.

$13 \mathrm{Vgl}$. Feit, E., a. a. O., S. $52 \mathrm{ff}$. 
Youth League sich immer unabhängiger von den Richtlinien der Zentralorganisation entwickelte ${ }^{14}$. Im Laufe der Zeit konnte die Youth League genügende Macht an sich bringen, daß die Besetzung des Präsidentenamtes des ANC im Grunde genommen von der Jugendbewegung diktiert wurde. Von 1949 ab lag die Kontrolle des Parteiapparats fest in Händen der Youth League. Auf ihr Verlangen wurde 1949 der Präsident Dr. Alfred Bitini Xuma gestürzt und der von der Jugendorganisation unterstützte Kandidat Dr. James S. Maroka an seine Stelle gesetzt. Nachdem Maroka die Erwartungen der Jugendbewegung nicht erfüllte, wurde er aus seinem Amt verdrängt und zum neuen Präsidenten Chief Luthuli gewählt. Im selben Jahr mußte die Parteiorganisation das radikale Aktionsprogramm der Jugendorganisation akzeptieren. Dieses Übernehmen des Aktionsprogrammes bedeutete eine strategische Abweichung von früheren Methoden - die sich auf die Bewilligung von Resolutionen und Memoranden beschränkten ${ }^{15}$.

\section{Die organisatorischen Realitäten}

Eine bloße Beschreibung der in der Verfassung stehenden Konzeptionen und Zielsetzungen gibt eigentlich nicht viel über die tatsächlichen Beziehungen zwischen den verschiedenen Gremien einer Organisation wieder. Zweifellos sahen die ANCFührer ihre Organisation als eine Einheit an, aber in der Tat war die Parteistruktur keineswegs ein einheitliches System. Vielmehr gab es mehrere einzelne Systeme, die unter getrennten Führungen standen, auch wenn sie mehr oder weniger gleiche Ziele verfolgten. Eine zentrale effektive Koordinationsinstanz existierte überhaupt nicht. Sicher war ein Konsensus über ein gewünschtes Ziel, nämlich die Gleichheit und das one-man-one-vote-Prinzip erreicht worden, jedoch blieben noch tiefliegende Unterschiede in wichtigen Dingen der Politik ungeklärt. Man muß mit Anthony Sampson übereinstimmen, wenn er der ANC als „the oddest and most mixed political body in the continent" 16 bezeichnet. Personen fast aller politischen Richtungen - Konservative, Anarchisten, Nationalisten, Kommunisten und Liberale - sind im ANC vorhanden. Die Zusammenfassung eines breiten politischen Spektrums von links bis rechts bedeutet notwendigerweise, daß keine einheitliche politische Richtung von den Anhängern und Mitgliedern erlangt werden kann. Es mag gewisse plausible Vorteile gegen politische Diskriminierungen geben, aber gerade für eine Opposition, der die parlamentarische Plattform versagt ist, ist die Entwicklung und klare Formulierung eines bestimmten "political ritual“ unausweichlich. Diese Unfähigkeit, bestimmte divergierende politische Richtungen auszuschließen, hat dazu geführt, daß die größte afrikanische politische Bewegung im Lande nicht in der Lage war, eine klare definitive politische Ideologie zu entwickeln. Für eine Bewegung, die das Ziel hat, die Massenunterstützung zu mobilisieren, bedeutete diese Tatsache einen ernsthaften Nachteil.

Außerdem führten der Mangel an finanziellen Mitteln und die nach geographischen Gesichtspunkten gebildeten Zweigorganisationen zur Zersplitterung. Die Kommunikation zwischen den Ortsgruppen und den Provinzzentralen wurde durch die weit ausgedehnten Entfernungen schwierig gemacht. Unvermeidlich hat diese Situa-

14 Für eine detaillierte Diskussion über die Entwicklung der Jugendbewegung siehe Benson, M., The African Patriots, London, 1963, S. $102 \mathrm{ff}$; vgl. Feit, E., a. a. O., S. 59.

15 Vgl. Nkoana, M., "Will we ever Learn?“ in: The New African, London, October, 1966.

16 Sampson, A., a. a. O., S. 41. 
tion dazu beigetragen, daß sich Zweigorganisationen zu selbständigen oder halbselbständigen Ortseinheiten entwickelten. Das Nichtvorhandensein von Parteizeitungen ist zu nennen. Wichtige Parteientscheidungen des Vorstandes wurden entweder verbal oder durch hektographierte Rundschreiben und durch andere ineffektive improvisierte Methoden den Zweigorganisationen bekanntgemacht - und dies in einem Polizeistaat mit einem außerordentlich effizienten Geheimdienst. Die Durchführung der getroffenen Entscheidungen wurde praktisch den Ortsparteiführungen ohne jede wirkliche Kontrolle überlassen. Da für jede politische Organisation (besonders für eine Massenbewegung in politisch-sozialen Verhältnissen wie in Südafrika), die Orts- und Zweigorganisationen den Grundstein der Organisation bilden oder bilden sollten, wäre zu erwarten, daß der ANC seine Ortsstruktur und seine Ortsmitgliedschaft zur wichtigsten Machtquelle entwickelt hätte. Die Schaffung und die ständige Aufsicht von Ortsgruppen hätte Priorität bekommen müssen. Die Expansion und die Durchorganisierung einer Partei verlangen eine ungebrochene nähere Bekanntschaft $\mathrm{zw}$ wschen der Führung und den Zweigstellen.

Nichtsdestoweniger war die Parteiführung schlecht informiert über die Zahl der Ortsgruppen und den Stand der Parteimitgliedschaft ${ }^{17}$. Jedoch wurden Bemühungen gemacht, die genaue Zahl und den Stand der Ortsgruppen festzustellen, dennoch blieben Briefe häufig unbeantwortet, so daß die Parteiführung sich auf Schätzungen verlassen mußte. Ortsgruppen traten auf und verschwanden, ohne daß der Vorstand davon Kenntnis bekam ${ }^{18}$.

Diese enorme Lücke zwischen der Führung und den Ortsstellen führte zu ernsthaften und zahlreichen Folgen. Die Selbständigkeit der Zweigorganisationen bedeutete, daß Ortsprobleme vor nationalen Issues Vorrang erhielten. Deshalb fanden es Ortsmitglieder nicht opportun, sich mit nationalen Problemen zu befassen. Wurden die nationalen Issues nicht mit dem direkten Anlaß der Umgebung verbunden, so war der Enthusiasmus für diese Probleme schwierig zu erwecken. Als beispielsweise die Provinz von Transvaal mit dem Problem des "pass law" konfrontiert wurde, gewährten die Ortsgruppen der Provinz Natal nur ungern ihre Unterstützung, da sie sich nicht bedroht fühlten ${ }^{19}$. Das Fehlen von Zentralkontrolle führte dazu, daß die Arbeitsweise der Ortsgruppen von der lokalen Führung entschieden wurde, und damit war die Wirksamkeit der Bewegung von der untersten Ebene der Partei abhängig. Erstaunlicherweise wurden immer die tüchtigen und fähigen Ortsfunktionäre in die Zentralparteiverwaltung aufgenommen, außer denjenigen, die ihre Nichtübereinstimmung mit dem Vorstand gezeigt hatten. Aber während der ANC innerhalb seiner Mitgliedschaft divergierende Ansichten billigte, wurden diese divergierenden Ansichten in höheren Entscheidungsgremien der Partei ausgeschlossen. Die Folgen waren Mißverständnisse und Bildung von zahlreichen Splitterorganisationen. Von Ortsgruppentätigkeiten war selten in afrikanischen oder weißen Zeitungen zu lesen. Die einzigen "feed back" Quellen (also die Ortsorganisationen selbst), sahen keinen Anlaß, über ihre Aktivitäten zu berichten. Aber wenn die Ortsgruppen der Provinzialorganisation nichts berichteten, so bedeutet dies, daß auch die Provinzen dem Hauptquartier keine genauen Berichte vorlegen konnten ${ }^{20}$. Der Vorstand hat jedoch versucht, die Parteiorganisation zu

\footnotetext{
$17 \mathrm{Vgl}$. Record of the South African Treason Trial, Regina vs. Farid Adams and Twenty-Nine Others, Luthuli, oral 11449.

$18 \mathrm{Vgl}$. Report of the ANC (Cape) Secretariat to the Provincial Conference, Cradock, August 1953.

19 Vgl. Mathews, Z. K., in the Treason Trial, a. a. O., 17980.

20 Vgl. Luthuli, Chief, in Ibid, oral 11448-49; 15800-802.
} 
reformieren. Diese Versuche blieben aber ohne Erfolg. Der sogenannte M-Plan, der die „Branch-method“ durch eine Umgruppierung der Bewegung nach Straßen-, Block- und Arbeitsstelleneinheiten ersetzen sollte, war einer dieser Renovationspläne Wegen der Kompliziertheit des Planes, aber auch zum Teil weil der Plan größere organisatorische Kosten verlangte, blieb es ein Vorschlag auf dem Papier ${ }^{21}$.

\section{Finanzen}

Finanziell betrachtet war der ANC eine arme Organisation. Edward Feit meint, daß diese Situation durch die mangelhafte Kommunikation zu erklären sei. Vielleicht gibt es aber noch tiefere Gründe, denn es ist nicht klar, warum z. B. afrikanische Parteien in Sambia und Malawi mit modernen Organisationsapparaten ausgerüstet sind, während eine noch besser ausgebildete afrikanische Führung in Südafrika nur eine "bumbling hand-to-hand"-Organisation einrichten konnte ${ }^{22}$. „You may perhaps be surprised to learn that ever since Conference last year, there have been no monies coming into this office. The bank balance of the national office is NIL . . ."23. Die finanziell chaotische Lage war Ergebnis der Tatsache, daß die Ortsgruppen nur geringe Beträge an die Zentralverwaltung schickten. Die Organisationen "were all guilty of using money that does not belong to them, that share which belongs to the province and national Headquarters"24.

Zur Klärung der finanziellen Situation soll auf folgendes hingewiesen werden: Der ANC-Jahresmitgliedsbeitrag betrug kaum mehr als 1,25 DM. Hinzu kamen kleine Sondersammlungen. Obgleich der ANC-Präsident erklärt hatte, daß viele Afrikaner es sich nicht leisten können, diese kleine Summe beizutragen, kann dieser Grund für die in den Städten lebende afrikanische Mehrheit nicht zutreffen. Immerhin müßte die ANC-Führung über die Zahlungsfähigkeit ihrer Gefolgschaft schon im klaren gewesen sein, bevor sie sich für diesen Betrag entschied. Wahrscheinlich hat Luthuli die Lage richtig beurteilt, als er sagte, daß die Afrikaner es nicht ganz verstanden haben, daß die Erlangung der Freiheit und Gleichheit mit gewissen Opfern verbunden ist, und daß persönliche Belohnungen nicht jede Protestaktion begleiten ${ }^{25}$. Eine weitere Erklärung für finanzielle Mängel scheint in der Natur der Bewegung selbst zu liegen. Wie es in vielen Massenbewegungen der Fall ist, appelliert eine kleine Gruppe von Aktivisten an die Massen nur zu den Zeitpunkten, wenn sie für direkte Aktionen gebraucht werden können. In den $Z$ wischenzeiten genießt die Führung völlige Bewegungsfreiheit bei der Formulierung der Politik, ohne daß die Menge der Mitglieder mitwirkt. Die Nachteile einer autoritären Parteistruktur treten besonders dann hervor, wenn diese Partei in der Gegenwart oder der nahen Zukunft keine Erfolge erzielen kann. Wird die Partei jedoch ständig verfolgt ohne jede Hoffnung auf Macht, und ist die Bestrafung durch die Regierung die einzige Folge der Mitgliedschaft, wie es in Südafrika der Fall ist, dann wird durch solch eine Situation die Entschlossenheit der Kernaktivisten gestärkt, die peripherische Mitgliedschaft jedoch wird beträchtlich geschwächt werden.

21 Report of the Secretariat to the 44th Annual Conference, ANC, 1956.

22 Vgl. Sampson, A., a. a. O., S. 42.

23 Treasurer-General of the ANC to Secretary-General, Brief y, 5. Mai 1955.

24 ANC (Cape) Secretariat Report, January-November, 1955.

25 Annual Report of the National Executive Committee to the 42nd Annual Conference of the ANC, 16-19 December, 1954. 


\section{Modelle der Strategie}

Seit seiner Gründung war der ANC die einzige Massenbewegung, die die Interessen der Schwarzen vertrat. Die Strategie des ANC wollte demgemäß nur die Unterstützung der Schwarzen gewinnen. Es gibt Kritiker, die sagen, der ANC sei rassisch orientiert, weil die Mitgliedschaft von Weißen in dieser Bewegung nicht zugelassen ist. Jedoch lehnt der ANC die Mitgliedschaft von Weißen nicht grundsätzlich ab, sondern nur als Mittel des Selbstschutzes gegen die Infiltration der Weißen. Übrigens diente das Prinzip der weißen Vorherrschaft als Anlaß zur Gründung des ANC. Daß der ANC sich auf afrikanische "public opinion“ konzentrierte, ergab sich von selbst ${ }^{26}$. Da er eine Partei war, die verschiedene politische Ansichten zusammenfaßte, stellte die Auswahl der Aktionsmethoden das gravierendste Problem des ANC dar. Auf der einen Seite standen konservative Elemente, die „nonviolence" befürworteten, um jegliches Blutvergießen zu vermeiden. Außerdem wies der Rechtsflügel der Partei auf die Bedeutung der geringen weißen Sympathie hin. Auf der anderen Seite drängten die jungen Mitglieder, insbesondere die Anhänger der Youth League, auf Annahme des afrikanischen Nationalismus und radikale Maßnahmen ${ }^{27}$.

\section{a) Passiver Widerstand}

Schließlich entschied man sich für eine Kompromißstrategie, um blutige Auseinandersetzungen zu vermeiden. $\mathrm{Da}$ der Wirtschaftsprozeß von der Arbeitskraft der Schwarzen abhängig ist, wurde der Streik als unmittelbare Waffe gewählt. Passive Widerstandsaktionen, wie Streiks, „stay-at-home“-Demonstrationen und der Boykott wurden häufig mit gewissem Erfolg angewendet. Aber größtenteils waren diese Erfolge reformistischer Natur, die hauptsächlich die Beziehungen zwischen Arbeitnehmer und Arbeitgeber regelten. Der Streit mit dem „Western Areas Tram Committee" und die Kontroverse mit der „Public Utility Transport Corporation“ sowie die Kampagne gegen den „Nursing Amendment Act“ sind Beispiele solcher Widerstandsaktionen reformistischen Charakters ${ }^{28}$.

Aber in Südafrika kann die Teilnahme an Streiks oder Demonstrationen harte Folgen haben. Die Teilnehmer solcher Veranstaltungen müssen damit rechnen, daß sie z. B. ihre Wohnräume verlieren, oder daß ihren Kindern die Möglichkeit zur Weiterausbildung entzogen wird. Der permanente Verlust einer Arbeitsstelle und die Vertreibung aus den Vorstädten in den Busch sind häufige Konsequenzen, die die Demonstranten in Kauf nehmen müssen. Außerdem ist es immer wieder vorgekommen, daß die Regierung administrative und juristische Anordnungen in Gang setzte, bevor der ANC die notwendige Aktionsorganisation vorbereiten konnte. So werden politische Streiks zur Sabotage erklärt, die mit der Todesstrafe für den Partizipanten bedroht ist. Regierungsmaßnahmen dieser Art sind durch den Gebrauch von Gesetzen wie z. B. des Masters and Servants Law (Transvaal and Natal Act), des Native Labour Settlement of Disputes Act und des Suppression of Communism Act durchaus im Rahmen des südafrikanischen Rechtswesens legitim. Ein Streik wird mit der Mobilisierung der Polizei und der Armee beantwortet.

26 Vgl. Sampson, A., a. a. O., S. 43.

27 Vgl. Benson, M., a. a. O., S. 157

28 Vgl. Mandela, N., Our Struggle Needs Many Tactics in: First, R., (ed.), a. a. O., S. 7 ff. 
Nelson Mandellas Versuch, im Jahre 1961 einen allgemeinen Streik zu organisieren, führte zu seiner Gefangennahme ${ }^{29}$. Damit war der Streikmöglichkeit ein Ende gemacht. Das bedeutete aber, daß dem ANC alle friedlichen legalen Mittel untersagt wurden. Endlich mußte die afrikanische Parteiführung ihre Augen öffnen und anerkennen, daß die Politik des "non-violence“ in einem System wie Südafrika keine Realpolitik war. "Who will deny", beklagte sich der ANC-Präsident und Friedens-Nobelpreisträger Luthuli, "that thirty years of my life have been spent knocking in vain, patiently, moderately and modestly at a closed and barred door? What have been the fruits of moderation? The past thity years have seen the greatest number of laws restricting our rights and progress until to-day we have reached a stage where we have almost no rights at all“30.

\section{b) Bewaffneter Kampf}

Grundprinzip der Aktionsstrategie afrikanischer Nationalisten ist jetzt eine Kombination von Gewalt und diplomatischer Aktion mit dem Ziel, a) die weiße „supremacy“ in Frage zu stellen, und b) Südafrika von der internationalen Gemeinschaft $\mathrm{zu}$ isolieren. $\mathrm{Da}$ seit 1960 afrikanische politische Organisationen verboten sind, operiert der ANC hauptsächlich als eine Untergrundbewegung. Colin Legum sagt, daß ein Organisationsnetz innerhalb Südafrikas existiert. Die Koordinatsfunktion wird durch die jungen "cadres" aufrechterhalten. Diese Menschen sind eine Generation von Studenten- und Gewerkschaftsleitern, Lehrern und Industriearbeitern mit organisatorischer Erfahrung. Ihre politischen Überzeugungen unterscheiden sich von denen der alten Eliten: kritisch gegenüber christlichen Werten, weniger kompromißbereit, militant und überzeugt, daß nur durch Kampf die Freiheit errungen werden kann. Colin Legum meint, daß es durch ein kompliziertes System dieser jungen „cadres“ gelungen ist, ständig Kontakt mit der im Gefängnis sitzenden Parteiführung zu halten.

Die Leitung der Untergrundaktivitäten ist jedoch zur Zeit von der Parteiführung in Exil übernommen worden, die ihr Hauptquartier in Dar es Salaam hat und über weitere Organisationsbüros in Lusaka (Sambia) und in London verfügt. Der ANC ist dadurch in die direkte Verbindung mit unabhängigen afrikanischen Staaten gebracht worden. Das African Liberation Committee (oder das Committee of Nine) mit Sitz in Dar es Salaam, eine Zweckorganisation der Organisation of African Unity (OAU), finanziert, bewaffnet und bildet die Untergrundbewegung aus, obgleich Unterstützung von Staaten außerhalb Afrikas nicht abgelehnt wurde. Das Ziel ist keineswegs eine direkte militärische Konfrontation der afrikanischen Staaten mit Südafrika, sondern eine Vorbereitung auf interne Auseinandersetzung nach dem Muster des Guerilla-Volkskrieges.

Die Einrichtung von Widerstandszentren außerhalb des Landes erlaubt es den Afrikanern Südafrikas, direkte Verbindung mit den anderen Freiheitsbewegungen von Angola, Mozambique, Südwestafrika und Rhodesien aufzunehmen. Da diese Länder Nachbarstaaten sind, sieht man es für notwendig und sinnvoll an, die noch unter weißer Herrschaft stehenden Gebiete als eine einzige. Einheit in politischer und militärischer Hinsicht zu betrachten. Diese Solidarität zwischen der MPLA von

29 Vgl. Legum, C. \& M., a. a. O., S. 193, vgl. Benson, M., a. a. O., S. 173.

30 Mandela, N., zitiert Ćhief Luthuli während des Rivonia-Prozesses am 20. 4. 1964, in: First, R. (ed.), 2. a. O., S. 165 . 
Angola, der ZAPU von Zimbabwe (Rhodesien), der FRELIMO von Mozambique, der SWAPO von Südwestafrika und dem ANC Südafrikas soll es den Beteiligten ermöglichen, einen effektiven Widerstand zu entwickeln ${ }^{31}$. Die Zusammensetzung der Widerstandskräfte hat schon eine defensive Raktion der Regierung Südafrikas hervorgerufen. Daß die Regierung sich mehr bedroht fühlt als zuvor, wird klar durch die Entsendung südafrikanischer Truppen nach Rhodesien, wo sie gemeinsam mit den rhodesischen Sicherheitseinheiten die Guerillakrieger an der rhodesischen Grenze zu Sambia bekämpfen. Uber hundert Afrikaner sind schon im ZambeziValley gefallen, während die portugiesische Luftwaffe ihre Aufgabe darin sieht, die kleinen Dörfer in Sambia zu terrorisieren ${ }^{32}$.

\section{Die Schwächen der ANC-Opposition}

An der Fortsetzung der europäischen Vorherrschaft in Südafrika haben zum großen Teil die Afrikaner selbst schuld. Obgleich die Regierung über enorme Machtmittel verfügt, findet sie keine Unterstützung durch die Schwarzen, die die Mehrheit der Bevölkerung bilden. Zunächst scheint es so zu sein, daß der ANC es versäumt hat, sich ein klares Bild über die eigene Macht im Verhältnis zu der der Regierung zu verschaffen. Das hat dazu geführt, daß der ANC große Projekte vorgenommen hat, über deren Ergebnis innerhalb der Parteileitung keine Sicherheit bestand. Die Ziele dieser Protestaktionen waren so allgemein formuliert, daß keine direkte Verbindung zu den Problemen der einfachen Afrikaner in den Reservaten und in den Vorstädten hergestellt wurde. In vielen Fällen, wie z. B. der „Western Areas Removal Campaign“ und der „Bantu Educational Campaign“, brachten diese Protestaktionen nicht das erwartete Ergebnis. All das führte zur allgemeinen Entmutigung der Parteianhänger. Zweitens soll der blinde Glaube an die Kraft des Boykotts und des passiven Widerstands als politische Waffe, um damit die Ansichten der Weißen zu ändern, genannt werden. Daß man trotz der harten Fakten jahrelang an die Stärke des passiven Widerstandes geglaubt hat, ist auffallend. Drittens scheint die Schwäche des ANC an Mängeln der personellen und materiellen Resourcen zu liegen. Die Regierung brauchte nur die wenigen Führungskräfte des ANC auszuschalten, um die afrikanische Partei in eine Position der Schwäche zu versetzen. Viertens: die Wertvorstellungen, die von dem ANC vertreten wurden, entfremdeten wichtige und zahlreiche Gruppen innerhalb der Partei. Das Predigen von "non-violence" und die Politik der Zusammenarbeit mit den anderen ethnischen Gruppen Südafrikas identifizierte die ANC-Führung mit bürgerlichen Werten, die der großen Zahl der jüngeren Mitglieder fremd sind. Eine wichtige Rolle beim Auseinanderfall der Emanzipationsbewegung spielte der ANC, indem er sich mit zahlreichen ethnischen Gruppen verbündete. Der sogenannte Volkskongreß, eine Dachorganisation der außer dem ANC auch der Congress of Democrats, der South African Congress, die South African Coloured Peoples Organisation und die südafrikanischen Gewerkschaften angehören, stellte für viele junge ANC-Mitglieder eine Herausforderung dar. Der ANC hatte keine Lehren aus der Entwicklung in Zentralafrika gezogen, wo die Inder mit ihren wirtschaftlichen Interessen die

31 Vgl. „Sechaba“, Offical Organ of the ANC, Vol. 1 No. 6, London, June 1967, S. 1 ff. Vgl. Nkoana, M., South Africa: The Big Question Mark for the OAU, in: The New African, London, October 1965, S. $185 \mathrm{ff}$

32 Siehe: The London Times, Rhodesia prepares for terrorist Attacks, I.ondon, November, 1968. 
Föderation von Rhodesien und Njassaland unterstützten. Im täglichen Leben behandelten die Inder die Afrikaner als Untergerodnete. Befremdlich erschien den Afrikanern auch die Aufnahme des vorwiegend aus Weißen zusammengesetzten Congress of Democrats in den Volkskongreß. Die Jungbewegung des ANC argumentierte, daß die Weißen (militant oder nicht-militant) von dieser Konstellation profitieren würden und sich niemals völlig mit der afrikanischen Sache identifizieren könnten.

Auf die Dauer bewirkte die Zusammenarbeit mit den Weißen eine Frustration der jungen Kämpfer. 1958 spalteten sie sich vom ANC ab und gründeten den PanAfrican Congress. Diese Organisation inszenierte eine militante Kampagne gegen die Paß-Gesetze. Es kam zu Demonstrationen und Aufständen im ganzen Land, die schließlich zum Sharpeville-Zwischenfall führten, bei dem zahlreiche Afrikaner von der südafrikanischen Polizei getötet wurden. Als Reaktion auf das militante Verhalten der Pan-Afrikanisten schwenkte der ANC unter Führung von Nelson Mandela auf eine ähnliche politische Linie ein. Doch im April 1960 wurden beide Organisationen verboten. Persönliche Rivalitäten in der Führungsspitze haben den Pan-African Congress praktisch gelähmt, so daß er heute nur noch auf dem Papier existiert. die cristlich-liberale Weltanschauung der Führer der ANC verhinderte eine Änderung ihrer Taktik. Führer der Partei erhielten ihre Ausbildung nur in Missionsschulen. Aus Pastoren wurden gleichzeitig Parteiführer. Schließlich muß die Struktur der Partei selbst erwähnt werden. Die Unfähigkeit der Parteiführung, ein operatives Kommunikationssystem zu entwickeln, das das „Learning“ innerhalb des Organisationsapparates ermöglichen würde, war Ursache zahlreicher irriger „self-defeating“-Entscheidungen, beispielsweise daß der ANC das Selbstverständnis einer Pressure Group entwickelte und nicht das einer revolutionären Organisation. Der ANC hoffte, daß sich durch göttliche Fügung die Dinge eines Tages zum Besseren kehren werden. Das Bestreben, innerhalb der Legalität zu bleiben, bedeutete die gleichzeitige Bereitschaft, sich einer Uberwachung zu unterstellen. Die Aktionen des ANC waren meistens nur Reaktionen auf Handlungen der Regierung. Im Laufe der Zeit verlor der ANC die Initiative. Als der ANC verboten wurde, gründete man die Untergrundorganisation. Der ANC war aber personell und organisatorisch auf eine Untergrundtätigkeit nicht vorbereitet.

Der Mißerfolg des ANC ist darauf zurückzuführen, daß es ihm nicht gelang, auf dem Lande effiziente Organisationsformen zu entwickeln. Durch die Schaffung der Batustane sicherte sich die Regierung die Einflußnahme auf die Häuptlinge und auf die Dorfbewohner. Kamuzu Banda und Jomo Kenyatta starteten ihren Feldzug auf dem Lande, weil die Mehrheit der Afrikaner in ländlichen Regionen lebt. Dort konnten sie sich organisieren, ohne befürchten zu müssen, daß die Regierung der Bevölkerung die Existenzgrundlage entziehen könnte. $\mathrm{Da}$ er die Basis auf dem Lande verloren hat, wird der ANC nur sehr schwer in der Lage sein, einen wirksamen Guerillakrieg zu inszenieren. Es ist völlig unmöglich, einen subversiven Krieg zu führen, ohne die Unterstützung der Dorfbevölkerung. Anstatt den Kampf im Busch aufzunehmen, unterhält der ANC kostspielige Public Relations Büros, die nicht den mindesten Beitrag zur Erreichung der Ziele des südafrikanischen Volkes leisten. All das reduziert erheblich die Effektivität der Partei, trotz der Unterstützung, die der ANC innerhalb der afrikanischen Gemeinschaft genießt. 